\title{
Young, metal-enriched cores in early-type dwarf galaxies in the Virgo cluster based on colour gradients
}

\author{
Linda Urich ${ }^{1}$, Thorsten Lisker ${ }^{1}$, Joachim Janz ${ }^{2,3}$, Glenn van de Ven ${ }^{4}$, Ryan Leaman ${ }^{4}$, Alessandro Boselli ${ }^{5}$, \\ Sanjaya Paudel ${ }^{6}$, Agnieszka Sybilska ${ }^{7}$, Reynier F. Peletier ${ }^{8}$, Mark den Brok ${ }^{9}$, Gerhard Hensler $^{10}$, Elisa Toloba ${ }^{11}$, \\ Jesús Falcón-Barroso ${ }^{12,13}$, and Sami-Matias Niemi ${ }^{14}$ \\ 1 Astronomisches Rechen-Institut, Zentrum für Astronomie der Universität Heidelberg, Mönchhofstraße 12-14, 69120 Heidelberg, \\ Germany \\ e-mail: linda@dwarfgalaxies.net \\ 2 Centre for Astrophysics and Supercomputing, Swinburne University, Hawthorn, VIC 3122, Australia \\ 3 Division of Astronomy, Department of Physics, University of Oulu, PO Box 3000, 90014 Oulun Yliopisto, Finland \\ ${ }^{4}$ Max-Planck-Institut für Astronomie, Königstuhl 17, 69117 Heidelberg, Germany \\ 5 Aix-Marseille Université, CNRS, LAM, Laboratoire d'Astrophysique de Marseille, 13388 Marseille, France \\ ${ }^{6}$ Department of Astronomy \& Center for Galaxy Evolution Research, Yonsei University, 03722 Seoul, Korea \\ 7 European Southern Observatory, Karl-Schwarzschild-Strasse 2, 85748 Garching bei München, Germany \\ 8 Kapteyn Astronomical Institute, University of Groningen, Postbus 800, 9700 AV Groningen, The Netherlands \\ 9 Institute for Astronomy, ETH Zurich, Wolfgang-Pauli-Strasse 27, 8093 Zurich, Switzerland \\ 10 Department of Astrophysics, University of Vienna, Tuerkenschanzstr. 17, 1180 Vienna, Austria \\ 11 University of the Pacific, Department of Physics, 3601 Pacific Avenue, Stockton, CA 95211, USA \\ 12 Instituto de Astrofísica de Canarias, C/ Via Láctea s/n, 38200 La Laguna, Canary Islands, Spain \\ 13 Departamento de Astrofísica, Universidad de La Laguna (ULL), 38206 La Laguna, Tenerife, Spain \\ 14 Mullard Space Science Laboratory, University College London, Holmbury St Mary, Dorking, Surrey RH5 6NT, UK
}

Received 30 March 2017 / Accepted 4 July 2017

\begin{abstract}
Early-type dwarf galaxies are not simply featureless, old objects, but were found to be much more diverse, hosting substructures and a variety of stellar population properties. To explore the stellar content of faint early-type galaxies, and to investigate in particular those with recent central star formation, we study colours and colour gradients within one effective radius in optical $(g-r)$ and near-infrared $(i-H)$ bands for 120 Virgo cluster early-type galaxies with $-19 \mathrm{mag}<M_{r}<-16$ mag. Twelve galaxies turn out to have blue cores, when defined as $g-r$ colour gradients larger than $0.10 \mathrm{mag} / R_{\mathrm{eff}}$, which represents the positive tail of the gradient distribution. For these galaxies, we find that they have the strongest age gradients, and that even outside the blue core, their mean stellar population is younger than the mean of ordinary faint early-type galaxies. The metallicity gradients of these blue-cored early-type dwarf galaxies are, however, in the range of most normal faint early-type galaxies, which we find to have non-zero gradients with higher central metallicity. The blue central regions are consistent with star formation activity within the last few $100 \mathrm{Myr}$. We discuss whether these galaxies could be explained by environmental quenching of star formation in the outer galaxy regions while the inner star formation activity continued.
\end{abstract}

Key words. galaxies: clusters: individual: Virgo - galaxies: dwarf - galaxies: evolution - galaxies: structure galaxies: stellar content - galaxies: photometry

\section{Introduction}

The most commonly occurring morphological type in galaxy clusters (Binggeli et al. 1985) are early-type dwarf galaxies, defined as diffuse objects with smooth regular morphology (Sandage \& Binggeli 1984), which usually goes along with paucity of gas, dust, and star formation. At absolute $B$ magnitudes brighter than -14 mag and up to the end of the dwarf range at $-18 \mathrm{mag}\left(10^{8} \lesssim M_{\text {stellar }} \lesssim 10^{10} M_{\odot}\right)$, early-type dwarf galaxies have typically been classified as dwarf ellipticals (dE) and dwarf lenticulars (dS0, Binggeli \& Cameron 1991) or alternatively as spheroidals (Sph, Kormendy et al. 2009), while they have been known as dwarf spheroidals (dSph) at fainter magnitudes (Grebel et al. 2003). Their abundance and low mass means that they are ideal probes for studying the influence of high-density environments on galaxy formation and evolution.
The Virgo cluster is the nearest high-density region and thus provides a perfect setting to investigate low-mass galaxies (e.g. Roberts et al. 2007; Boselli et al. 2008, 2014; Toloba et al. 2015; Liu et al. 2016; Ferrarese et al. 2016). Furthermore, Virgo is dynamically young and field galaxies and groups of galaxies are falling into the cluster (e.g. Tully \& Shaya 1984; Binggeli et al. 1987; Kim et al. 2016).

While at first glance, most early-type dwarfs are small featureless objects, closer investigation has revealed that they can host substructures like weak spiral arms, bars, disks, nuclei, or blue cores (e.g. Jerjen et al. 2000; Barazza et al. 2002; De Rijcke et al. 2003; Lisker et al. 2006a,b, 2007, 2008; Janz et al. 2012). These features have stirred the debate about their origin and evolution. Given the scarcity of early-type dwarfs in the field (Binggeli et al. 1988), most proposed mechanisms rely on the idea of environmental transformation of late 
types. These include galaxy harassment caused by tidal forces from the overall cluster potential combined with occasional close high-speed encounters (Moore et al. 1996, 1998; Smith et al. 2010, 2015), as well as ram pressure stripping (Gunn \& Gott 1972; Van Zee et al. 2004a) and galaxy starvation (Larson et al. 1980).

Since it is debatable whether high-mass and low-mass early-type galaxies follow common scaling relations, it remains unclear whether continuously varying contributions of the same internal and external processes are sufficient to understand the properties of present-day early-type galaxies across a wide mass range (e.g. Graham \& Guzmán 2003; Janz \& Lisker 2008; Dabringhausen et al. 2008; Kormendy et al. 2009; Glass et al. 2011; Dabringhausen \& Kroupa 2013; Lisker et al. 2013; Sybilska et al. 2017; Janz et al. 2017). Traditionally, faint earlytype galaxies were separated into dwarf and normal early-types, with the term "dwarf" applied to the (visually) more diffuse galaxies (Sandage \& Binggeli 1984), which typically also have a shallower surface brightness profile (Binggeli \& Cameron 1991). At a given luminosity, this classification meant that a dwarf would have a larger effective radius than a non-dwarf galaxy. Since, however, the size-magnitude diagram does not show a gap between dwarfs and non-dwarfs, but instead an overlap, the SMAKCED ${ }^{1}$ collaboration decided to target faint early-type galaxies regardless of their size and their previous classification as (non-)dwarf (Janz et al. 2012, 2014). Since our sample is based on SMAKCED, we follow the same approach as presented in their study in order to avoid any subjectivity in selecting galaxies. Furthermore, analyses of spectroscopy for small samples of galaxies show no dichotomy in the stellar population properties or in the angular momentum content between faint early-type galaxies classified previously as dwarfs and non-dwarfs (Guérou et al. 2015; Toloba et al. 2014a).

Spectroscopic stellar population analyses of galaxies are usually preferred over photometry, since the stellar population properties are encoded in a number of spectral features (Vazdekis et al. 2012). They are, however, often limited to small sample sizes and inner galaxy regions (e.g. Paudel et al. 2011; Toloba et al. 2014b), due to the long exposure times required for spectroscopy of faint galaxies. Photometric studies investigating broad-band colours have the advantage of comprising larger numbers of objects and reaching larger radii even for more diffuse galaxies (e.g. Den Brok et al. 2011; Janz et al. 2014). Although they offer less detailed information for individual galaxies, they are still able to reveal information about the stellar ages and metallicities of galaxy populations (e.g. Schombert 2016; Roediger et al. 2017). Therefore, they provide a fundamental source for investigating their star formation and enrichment histories (e.g. Barazza et al. 2006; Kim et al. 2010) in connection with their local and large-scale environment (Barazza et al. 2009; Lietzen et al. 2012; Poudel et al. 2016, 2017). Galaxy colour gradients contribute additional information (see e.g. Peletier 1993; Pierini 2002), thereby helping to understand galaxy evolution. However, Roediger et al. (2011) found no systematic dependence of the stellar population gradients of Virgo cluster galaxies on environment. For disk galaxies, they argue that those with positive age gradients may have had their outer gas disks stripped off, while those with negative age gradients have likely grown inside-out. For early-type dwarfs, Roediger et al. pointed out that they seem incompatible with a

\footnotetext{
1 Stellar content, MAss and Kinematics of Cluster Early-type Dwarfs, http://www. smakced.net
}

primordial origin given their mostly positive age and negative metallicity gradients.

A particularly intriguing subclass of early-type dwarfs are those galaxies that possess young central stellar populations (Vigroux et al. 1984). They have been found in nearly all environments, from virtual isolation ( $\mathrm{Gu}$ et al. 2006) to galaxy groups (Hodge 1973; Tully \& Trentham 2008) and clusters (De Rijcke et al. 2003; Lisker et al. 2006b), in some cases even dominating the early-type dwarf population (Pak et al. 2014, Ursa Major cluster). Only in the massive Coma cluster were they found to be nearly absent (Den Brok et al. 2011). While Lisker et al. (2006b) defined the population of Virgo cluster blue-cored early-type dwarfs through visual inspection of $g-i$ colour maps, we present here a more quantitative analysis that makes use of the available optical and near-infrared imaging data.

This paper is organized as follows. In Sect. 2 we give an overview of our sample. Our analysis is described in Sect. 3. In Sect. 4 the resulting colours, colour gradients and their correlations are shown and separated into age- and metallicitysensitive colours. The interpretation and discussion of our results in terms of stellar populations and galaxy evolution are presented in Sect. 5.

\section{Sample and data}

We investigated a sample of 120 faint early-type galaxies in the Virgo cluster that span a luminosity range of -19 mag < $M_{r}<-16$ mag. Of these galaxies, 97 were classified as dwarf ellipticals and dwarf lenticulars and the other 23 galaxies were classified as normal (non-dwarf) ellipticals and lenticulars. This classification is based on the Virgo Cluster Catalog (VCC, Binggeli et al. 1985), and takes into account the updated membership by heliocentric velocities from Lisker et al. (2013). Our sample, based on Janz et al. (2014, SMAKCED), comprises $90 \%$ of Virgo cluster early-type galaxies within -19 mag $<M_{r}<-17 \mathrm{mag}$, and 50\% within $-17 \mathrm{mag}<M_{r}<-16 \mathrm{mag}$. Within the faintest $\sim 0.5$ mag of our sample's magnitude range, we are biased towards objects with brighter-than-average surface brightness. Our sample covers 14 of the 23 blue-cored early-type dwarf galaxies that Lisker et al. (2006b) identified qualitatively from colour maps.

Optical $g_{-}, r$-, and $i$-band images (background-subtracted, flux-calibrated, and astrometrically aligned) of our sample galaxies were provided by Lisker et al. (2007), based on the imaging data of the Sloan Digital Sky Survey (SDSS) Data Release 4 and Data Release 5 (Adelman-McCarthy et al. 2006, 2007), with a pixel scale of 0 '.396. Publicly available ${ }^{2}$ nearinfrared $H$-band images (background-subtracted and fluxcalibrated) from SMAKCED, obtained with the ESO New Technology Telescope (NTT), the Telescopio Nazionale Galileo, and the Nordic Optical Telescope, were provided by Janz et al. (2014), with a pixel scale of $0^{\prime \prime} .25$ and a median seeing of $0^{\prime \prime} .9$. Both data sets were corrected for Galactic extinction according to Schlegel et al. (1998).

In addition to the image treatment described in Lisker et al. (2007) and Janz et al. (2014) we had to align the $H$-band images with the optical images before measuring colours and colour gradients. Therefore, we changed the pixel scale of the $H$-band images with $\mathrm{IRAF}^{3} /$ magnify to 0 '.396 and aligned the $H$-band with IRAF/geomap and IRAF/geotran, feeding these tasks a

\footnotetext{
http://dc.zah. uni-heidelberg.de/browse/smakced/q Image Reduction and Analysis Facility, http://iraf.noao.edu/
} 


\section{VCC1501}

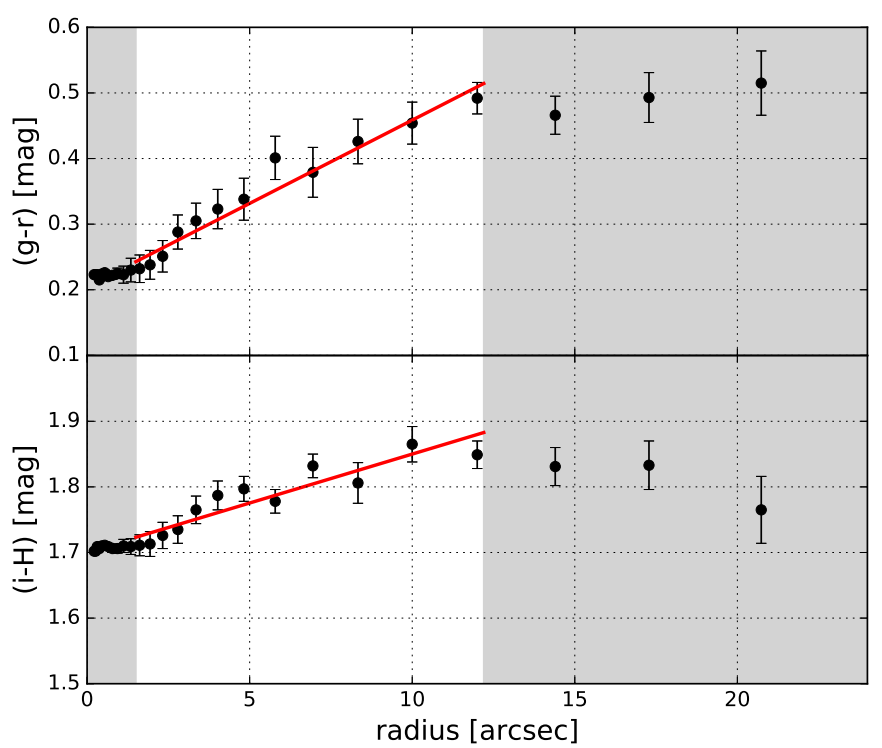

Fig. 1. Radial colour profile in $g-r$ and $i-H$ for the galaxy VCC 1501 . The $g-r$ colour (upper panel) and the $i-H$ colour (lower panel) are plotted against the radius of the galaxy. Linear gradients (red) were fitted from $1^{\prime \prime} 5$ to the effective radius (12"), taking into account the uncertainties of the isophotal colours. The resulting gradient values are $\delta(g-r)=0.305 \pm 0.027 \mathrm{mag} / R_{\mathrm{eff}}$ and $\delta(i-H)=0.179 \pm 0.023 \mathrm{mag} / R_{\mathrm{eff}}$. The area not used for the fit is shaded in grey.

list of objects in each image that was obtained with SExtractor (Bertin \& Arnouts 1996) ${ }^{4}$, and visually inspecting the result.

In order to take into account differences in the point spread functions (PSF) of the different bands to the first order, we measured the full width at half maximum (FWHM) of several stars for each wavelength band with SExtractor and convolved the respective better image of each pair of bands $(g \& r$ and $i \& H)$ with a Gaussian kernel with IRAF/gauss, using the quadratic difference of the PSF FWHM values and dividing it by 2.35 to yield the Gaussian $\sigma$. This yields a median FWHM in $g$ and $r$ of $1^{\prime \prime}$ ? with a $\pm 40 \%$ range of $\left[1{ }^{\prime \prime} 0,11^{\prime \prime} 6\right]$, as well as a median FWHM in $i$ and $H$ of $1^{\prime \prime}, 0$ and $\pm 40 \%$ range of $\left[0 \prime \prime 8,1^{\prime \prime} .3\right]$.

\section{Analysis}

To extract radial colour profiles in $g-r$ and $i-H$ we measured the average intensity in each band in about 20-30 elliptical annuli for each galaxy with IRAF/ellipse between the centre and the two effective radii. We use the semi-major axis of the halflight aperture in $H$ (published by Janz et al. $2014^{2}$ ) as effective radius. The semi-major axis of the elliptical annuli increases by $20 \%$ from one to the next. While running IRAF/ellipse we kept fixed the centre, the position angle and the ellipticity (measured in $r$ at the effective radius) of the galaxy. This defines a radial colour profile for each galaxy in both $g-r$ and $i-H$. We then fitted a weighted linear regression to the radial profiles using the non-linear least-squares Marquardt-Levenberg algorithm and taking into account the uncertainties of the isophotal colours with a weight of $1 / \sigma^{2}$, where $\sigma$ is the standard deviation of the respective colour value. We limited the range of our fits to radial bins with centres from 1 "'5 up to one effective radius since the median seeing of the SDSS $r$-band images is $1{ }^{\prime \prime} 4$ (Adelman-McCarthy et al. 2006, 2007). We decided not to use

\footnotetext{
4 http://www.astromatic.net/software/sextractor
}

the innermost colour values despite our approximate homogenization of the PSFs of different bands. We made this decision because the specific PSF shape at the position of the galaxy centre may not be accurately reflected by a simple Gaussian; in addition, stellar nuclei can lead to rather steep central intensity profiles, thus affecting central galaxy colours in case of PSF differences. The outer limit of our fit is set by the desire for a high signal-to-noise ratio, i.e. small statistical uncertainties, and by a visual inspection of the colour profiles which shows that profiles beyond the effective radius are mostly flat or very shallow (consistent with Lisker et al. 2006b), whereas the main interest of our study are objects with strong gradients.

This gives us quantitative radial colour gradients, defined by their slopes $\mathrm{d}(g-r) / \mathrm{d}\left(R / R_{\mathrm{eff}}\right)$ and $\mathrm{d}(i-H) / \mathrm{d}\left(R / R_{\text {eff }}\right)$, and parameter errors achieved by the fit, which are calculated as standard errors of a linear least-squares problem. In addition to these fitting uncertainties, we quantify the goodness of fit with the coefficient of determination, $\mathcal{R}^{2}$, on a scale from 0 to 1 (the larger, the better). The value of $\mathcal{R}^{2}$ is provided in Table 1 and helps to indicate how well our chosen linear fit is able to approximate a galaxy's colour profile. An example is provided in Fig. 1, which shows the $g-r$ and $i-H$ radial profile of the galaxy VCC 1501 and its fitted linear slopes from $11^{\prime \prime 5}$ to the effective radius, $12^{\prime \prime}$. This galaxy has one of the most extreme colour gradients in our sample: $\delta(g-r)=0.305 \pm 0.027 \mathrm{mag} / R_{\mathrm{eff}}\left(\mathcal{R}_{\delta(g-r)}^{2}=0.95\right)$ and $\delta(i-H)=0.179 \pm 0.023 \mathrm{mag} / R_{\mathrm{eff}}\left(\mathcal{R}_{\delta(i-H)}^{2}=0.85\right)$.

Since the existence of colour gradients complicates the definition of a global galaxy colour, we decided to choose the colour at the effective radius as representative of the main body, which avoids the contamination due to blue centres if we were to use radially integrated colours. In order to take the colour-magnitude relation of early-type dwarfs (Lisker et al. 2008) into account, we computed residual colours as follows. For a given galaxy with absolute magnitude $M_{r}$, we calculated the mean colour of all galaxies that lie in a magnitude interval of $M_{r} \pm 0.5 \mathrm{mag}$. The difference between the galaxy's colour value and the mean colour is its residual colour. For example, galaxy VCC 1488 has a $g-r$ colour of $0.52 \mathrm{mag}$ at the effective radius and an absolute magnitude of $M_{r}=-17.0 \mathrm{mag}$. The mean colour $\langle(g-r)\rangle$ of all galaxies in the magnitude interval $-17.5 \mathrm{mag}<M_{r}<-16.5 \mathrm{mag}$ is $0.62 \mathrm{mag}$ and hence the residual $g-r$ colour of VCC 1488 is $-0.10 \mathrm{mag}$.

\section{Results}

\subsection{Colours and colour gradients}

Our analysis yields linear $g-r$ and $i-H$ gradients for 120 faint early-type galaxies in the Virgo cluster (Fig. 2). There is no systematic difference between the colour gradients of galaxies originally classified as dwarfs and as non-dwarfs in Binggeli et al. (1985, also see Sect. 1).

We find slightly larger $g-r$ gradients for larger $i-H$ gradients and, while most $i-H$ gradients are negative (median: $\left.-0.116 \mathrm{mag} / R_{\text {eff }}\right), g-r$ gradients are centred around zero with the median at $-0.006 \mathrm{mag} / R_{\text {eff }}$. Additionally, we find a tail of galaxies having clearly larger $g-r$ gradient values. We thus define all 12 galaxies $^{5}$ with gradient values larger than $1 \sigma$ above the mean $\left(\langle\delta(g-r)\rangle=0.014 \mathrm{mag} / R_{\text {eff }}\right)$, i.e. with values above $0.10 \mathrm{mag} / R_{\text {eff }}$, as quantitative blue-cored

5 VCC 0021, VCC 0173, VCC 0209, VCC 0870, VCC 0951, VCC 1488, VCC 1499, VCC 1501, VCC 1512, VCC 1684, VCC 1779 , and VCC 1912. 
Table 1. Properties of quantitative blue-cored early-type dwarf galaxies.

\begin{tabular}{ccccccccccc}
\hline \hline $\begin{array}{c}\text { Object } \\
\text { VCC }\end{array}$ & $\begin{array}{c}M_{r} \\
{[\mathrm{mag}]}\end{array}$ & $\begin{array}{c}R_{\text {eff }} \\
{[\mathrm{arcsec}]}\end{array}$ & $\begin{array}{c}\delta(g-r) \\
{\left[\mathrm{mag} / R_{\mathrm{eff}}\right]}\end{array}$ & $\begin{array}{c}\mathcal{R}_{\delta(g-r)}^{2} \\
\begin{array}{c}\delta(i-H) \\
{\left[\mathrm{mag} / R_{\mathrm{eff}}\right]}\end{array}\end{array}$ & $\begin{array}{c}\mathcal{R}_{\delta(i-H)}^{2} \\
(g-r)_{1^{\prime \prime 5}} \\
{[\mathrm{mag}]}\end{array}$ & $\begin{array}{c}(i-H)_{1^{\prime \prime 5}} \\
{[\mathrm{mag}]}\end{array}$ & $\begin{array}{c}(g-r)_{R_{\mathrm{eff}}} \\
{[\mathrm{mag}]}\end{array}$ & $\begin{array}{c}(i-H)_{R_{\mathrm{eff}}} \\
{[\mathrm{mag}]}\end{array}$ \\
\hline 0021 & -17.4 & 14.2 & $0.22 \pm 0.03$ & 0.90 & $0.00 \pm 0.03$ & 0.00 & $0.33 \pm 0.02$ & $1.83 \pm 0.02$ & $0.50 \pm 0.03$ & $1.83 \pm 0.02$ \\
0173 & -16.9 & 14.5 & $0.15 \pm 0.02$ & 0.45 & $0.03 \pm 0.01$ & 0.24 & $0.39 \pm 0.02$ & $1.79 \pm 0.02$ & $0.57 \pm 0.02$ & $1.82 \pm 0.01$ \\
0209 & -16.9 & 16.9 & $0.11 \pm 0.04$ & 0.88 & $-0.34 \pm 0.04$ & 0.88 & $0.53 \pm 0.06$ & $2.06 \pm 0.06$ & $0.62 \pm 0.04$ & $1.81 \pm 0.06$ \\
0870 & -17.0 & 16.7 & $0.25 \pm 0.03$ & 0.91 & $0.00 \pm 0.03$ & 0.02 & $0.36 \pm 0.04$ & $1.81 \pm 0.04$ & $0.57 \pm 0.03$ & $1.82 \pm 0.03$ \\
0951 & -17.8 & 19.3 & $0.19 \pm 0.02$ & 0.88 & $-0.02 \pm 0.02$ & 0.03 & $0.45 \pm 0.02$ & $1.83 \pm 0.02$ & $0.58 \pm 0.02$ & $1.78 \pm 0.02$ \\
1488 & -17.0 & 14.0 & $0.19 \pm 0.02$ & 0.84 & $0.01 \pm 0.01$ & 0.01 & $0.37 \pm 0.01$ & $1.88 \pm 0.01$ & $0.52 \pm 0.02$ & $1.88 \pm 0.02$ \\
1499 & -16.8 & 7.5 & $0.29 \pm 0.02$ & 0.99 & $0.08 \pm 0.02$ & 0.68 & $0.16 \pm 0.01$ & $1.65 \pm 0.01$ & $0.39 \pm 0.02$ & $1.71 \pm 0.02$ \\
1501 & -16.3 & 12.0 & $0.31 \pm 0.03$ & 0.95 & $0.18 \pm 0.02$ & 0.85 & $0.23 \pm 0.02$ & $1.71 \pm 0.02$ & $0.49 \pm 0.02$ & $1.85 \pm 0.02$ \\
1512 & -16.5 & 12.1 & $0.21 \pm 0.03$ & 0.52 & $-0.12 \pm 0.03$ & 0.82 & $0.40 \pm 0.04$ & $1.94 \pm 0.04$ & $0.67 \pm 0.03$ & $1.83 \pm 0.02$ \\
1684 & -16.7 & 18.5 & $0.38 \pm 0.02$ & 0.88 & $-0.02 \pm 0.02$ & 0.05 & $0.20 \pm 0.02$ & $1.77 \pm 0.02$ & $0.50 \pm 0.02$ & $1.75 \pm 0.02$ \\
1779 & -16.9 & 19.6 & $0.20 \pm 0.02$ & 0.76 & $-0.17 \pm 0.02$ & 0.92 & $0.36 \pm 0.02$ & $1.86 \pm 0.03$ & $0.55 \pm 0.02$ & $1.69 \pm 0.02$ \\
1912 & -17.9 & 22.2 & $0.13 \pm 0.02$ & 0.86 & $0.02 \pm 0.03$ & 0.21 & $0.46 \pm 0.09$ & $1.90 \pm 0.10$ & $0.60 \pm 0.01$ & $1.93 \pm 0.02$ \\
\hline
\end{tabular}

Notes. The table lists: (1) designation; (2) absolute $r$-band magnitude; (3) effective radius (semi-major axis of half-light aperture in $H$ ); (4) $g-r$ gradient fitted from 1 ".5 to $R_{\text {eff }} ;$ (5) coefficient of determination for $\delta(g-r) ;(6) i-H$ gradient fitted from 1 '.5 to $R_{\text {eff }}$; (7) coefficient of determination for $\delta(g-r)$; (8) $g-r$ colour measured at 1 '.5; (9) $i-H$ colour measured at 1 '.5; (10) $g-r$ colour measured at $R_{\mathrm{eff}}$; (11) $i-H$ colour measured at $R_{\text {eff }}$.

early-type dwarf galaxies (indicated by the black horizontal tick in Fig. 2). Their colours and colour gradients are listed in Table 1. All but one of them (VCC 0209) were already classified as blue-cored early-type dwarfs by the qualitative investigation of Lisker et al. (2006b), which was based on visual inspection of $g-i$ colour maps. Lisker et al. (2006b) categorized four more early-type dwarfs from our sample as blue-cored (VCC 0170, VCC 0218, VCC 0308, and VCC 0781), which we find to have clearly smaller $g-r$ gradients. It needs to be noted, however, that VCC 0781 is found to have dust in its centre (di Serego Alighieri et al. 2013), which might affect the inner colours. For all 12 quantitative blue-cored early-type dwarf galaxies, and for the four additional objects in the Lisker et al. sample, we show the radial colour profile in Fig. 3.

For further investigation we divide our sample into four subsamples based on their $g-r$ gradients, such that each subsample includes 30 galaxies, one quarter of the whole sample. The ranges of these subsamples are shown by the colour-coding in Fig. 2. The blue subsample includes the galaxies with the largest, or strongest positive, $g-r$ gradients (bluer centre, redder outskirts), which is the highest quartile, whereas the red subsample includes the galaxies with the smallest, or strongest negative, $g-r$ gradients, which is the lowest quartile.

With quantitative colour gradients of faint early-type galaxies at hand, we can address the question of whether gradients are correlated with colours measured at the effective radius and/or the galaxy centre. In the histogram in Fig. 4 (top panel) the distribution of the $(g-r)_{R_{\text {eff }}}$ residual colour is shown for each of the four subsamples. In addition to the four above-mentioned subsamples, an additional one comprises only the quantitative bluecored galaxies (denoted "bc" and shown in black in the figure). We find that the blue-cored galaxies tend to have bluer $g-r$ residual colours at the effective radius and therefore even bluer $g-r$ residual colours in the centre than to the rest of the faint early-type galaxies.

A similar relation is seen in Fig. 4, middle panel, where the distribution of the $(i-H)_{R_{\text {eff }}}$ residual colour is displayed for the different $g-r$ gradient subsamples. Galaxies with quantitative blue cores tend to have bluer $i-H$ resdiual colours at the effective radius. Owing to the slight correlation between $g-r$ and $i-H$ gradients (Fig. 4, lower panel), the blue-cored galaxies also have

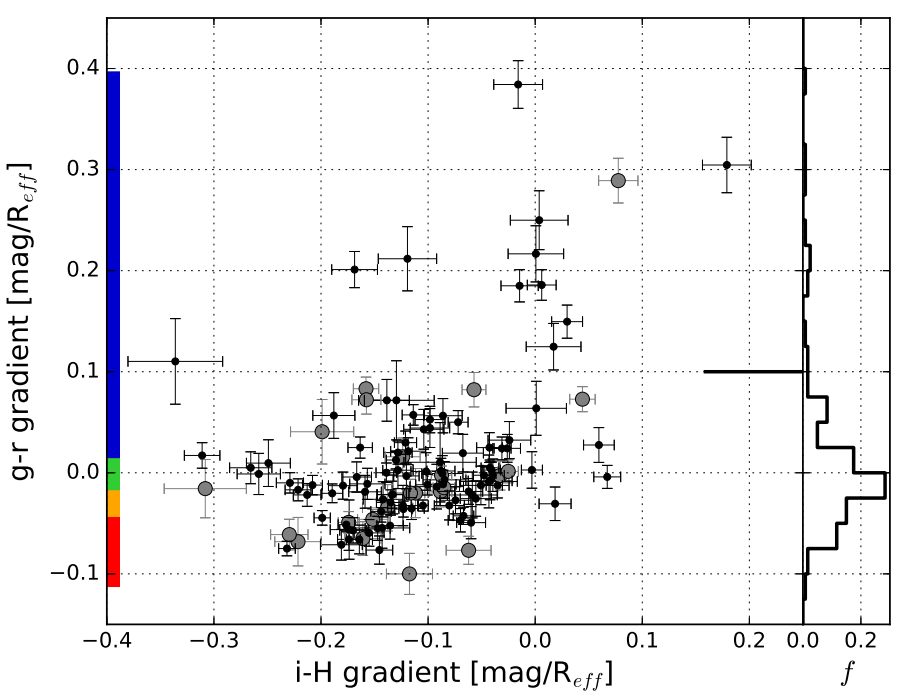

Fig. 2. Fitted $i-H$ gradients plotted against the fitted $g-r$ gradients. The error bars denote the standard errors (see text). Galaxies with $g-r$ gradients larger than $0.10 \mathrm{mag} / R_{\text {eff }}$ are defined as quantitative blue-cored early-type dwarf galaxies and are located above the black horizontal tick in the plot. Galaxies that were classified as non-dwarfs by Binggeli et al. (1985) are displayed as filled grey circles. The colour-coding on the $y$ axis defines the ranges of four subsamples that all include the same number of galaxies (30). The fraction $f$ of galaxies in the $g-r$ gradient distribution is presented in the right histogram.

systematically bluer $i-H$ residual colours at the centre with respect to the other galaxies of our sample.

\subsection{From colours to stellar populations}

Interpreting our results leads us to the issue of separating age and metallicity effects. It is well known that young stellar populations include hot, massive, short-lived stars that lead to a bluer integrated colour and, in contrast, that a high metallicity content leads to a redder colour. Therefore, we have an age-colour relation and a metallicity-colour relation, which are both relevant for the interpretation of stellar populations. The question is how strongly each relation contributes to a given observed 
L. Urich et al.: Young, metal-enriched cores in early-type dwarf galaxies

\section{A: Quantitative blue-cored early-type dwarf galaxies}
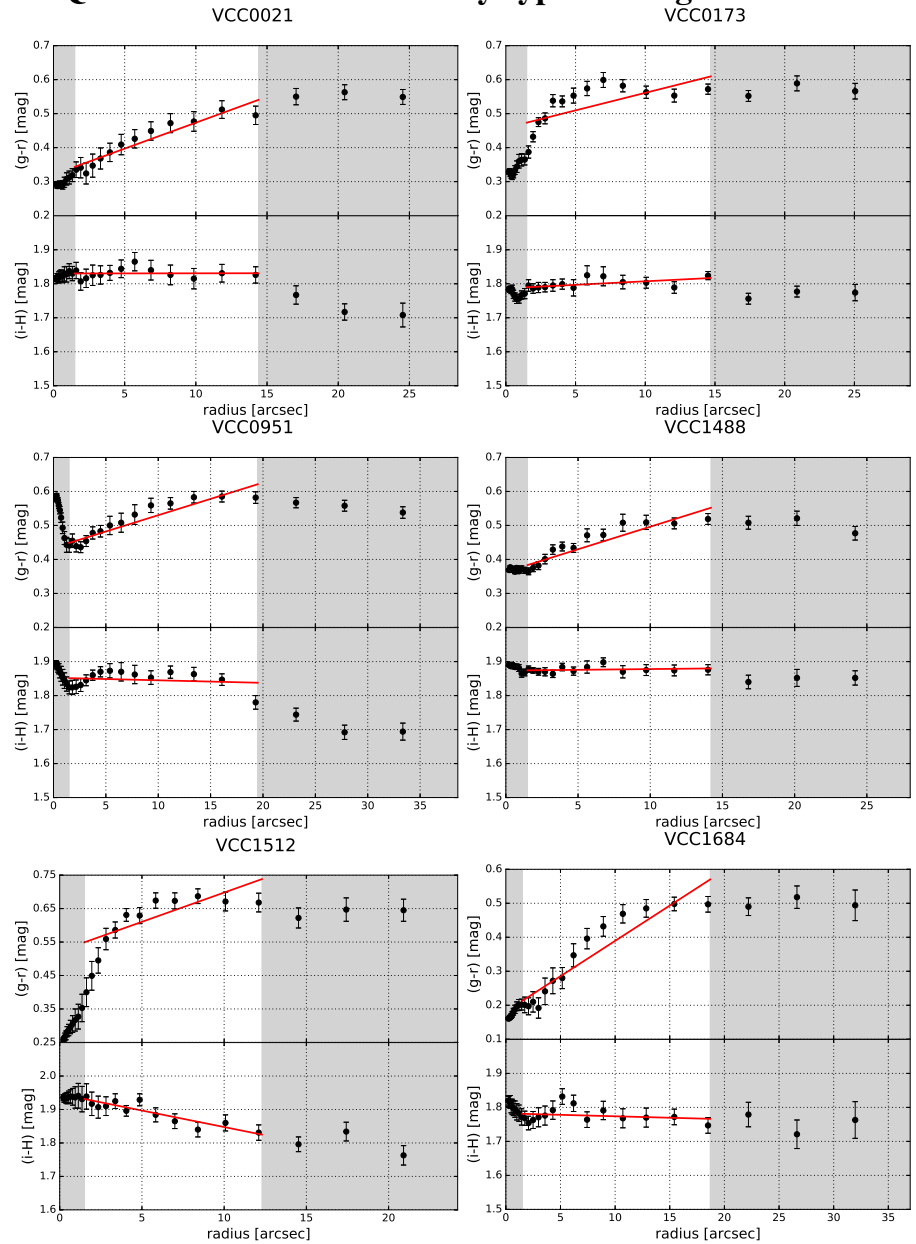
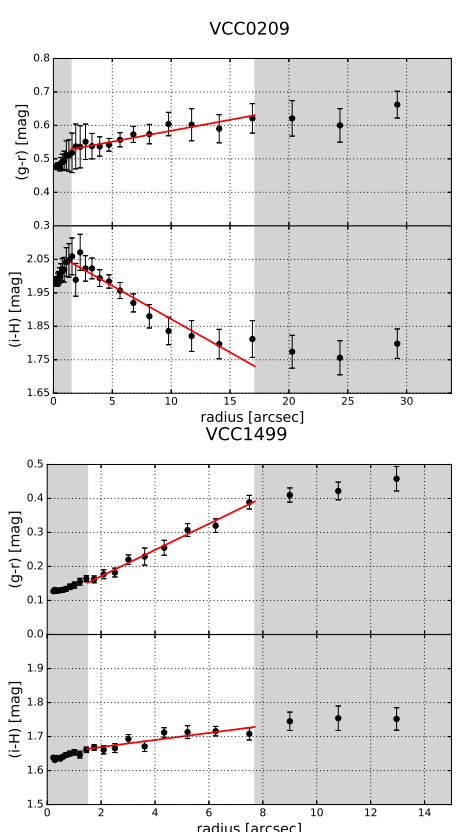

radius $[$ arcsec]
VCC1779

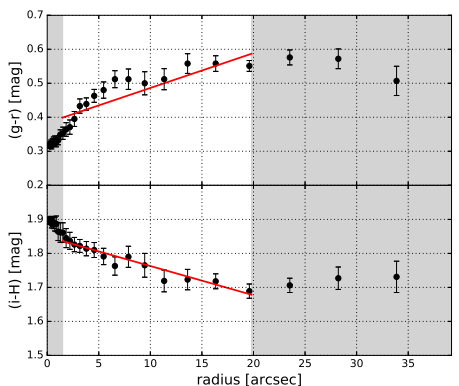

vccos70

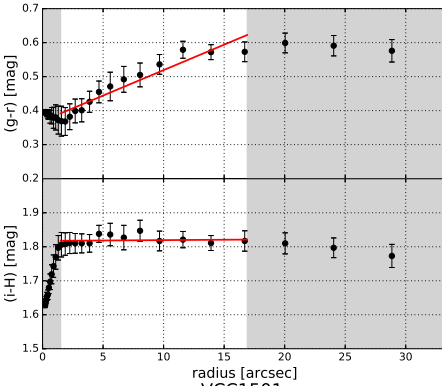

radius [arcsec]

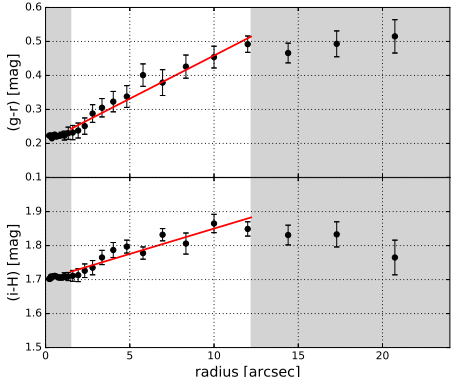

radius $[\operatorname{arcsec}]$
VCC1912

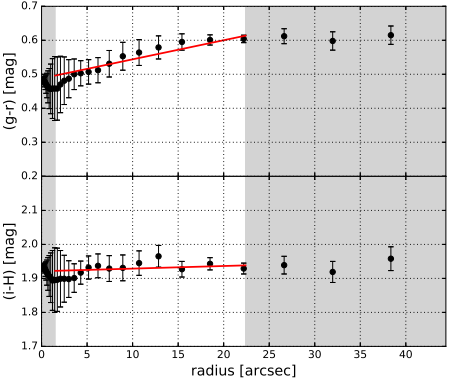

\section{B: No quantitative blue-cored early-type dwarf galaxies}
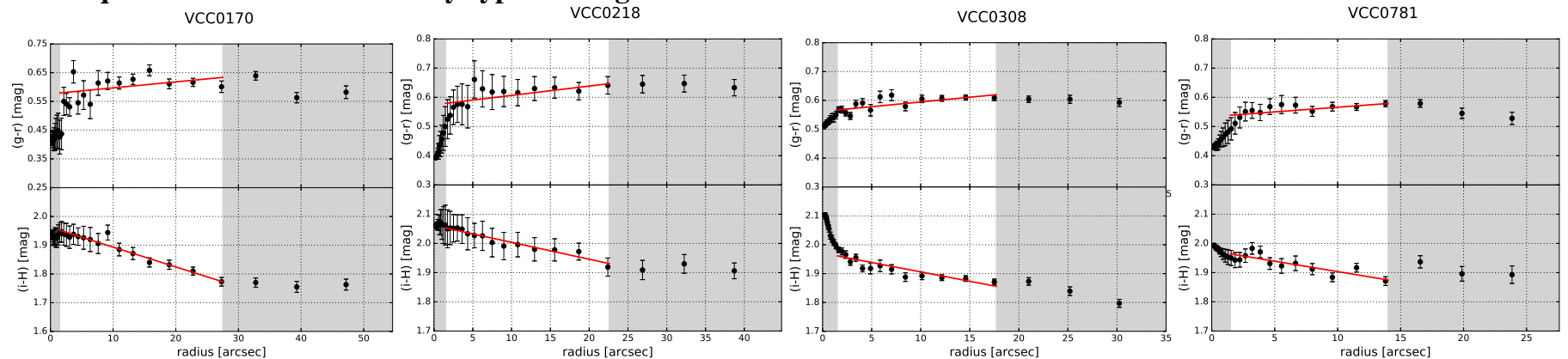

\section{C: Normal early-type dwarf galaxies}
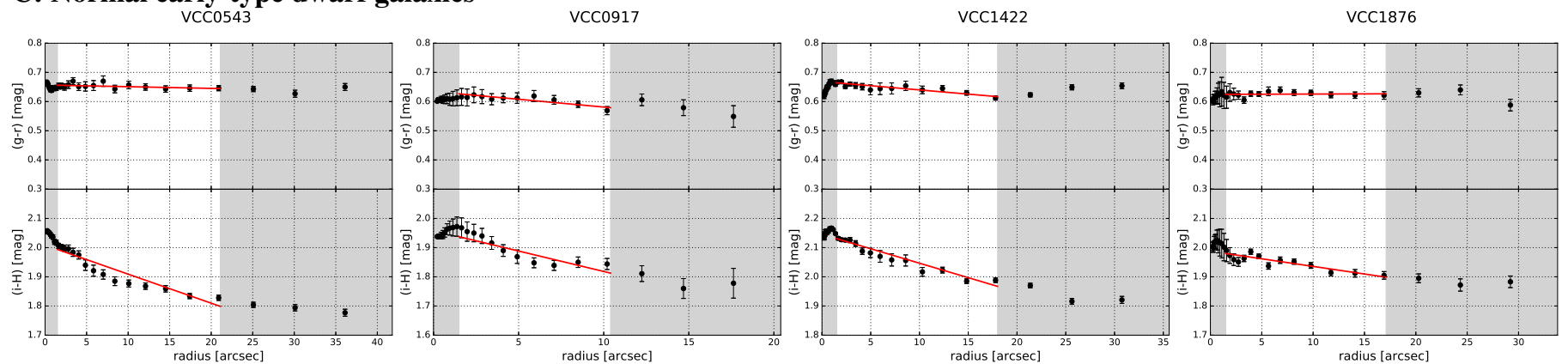

Fig. 3. $A$ : radial colour profiles in $g-r$ and $i-H$ (same as in Fig. 1) for all 12 quantitative blue-cored ( $g-r$ gradient $>0.10$ mag $\left./ R_{\text {eff }}\right)$ early-type dwarf galaxies. $B$ : radial colour profiles of the four galaxies that were classified as qualitative blue-cored early-type dwarf galaxies by Lisker et al. (2006b), but which we found to have clearly smaller $g-r$ gradients than $0.10 \mathrm{mag} / R_{\mathrm{eff}} . C$ : four normal early-type dwarfs for comparison. 

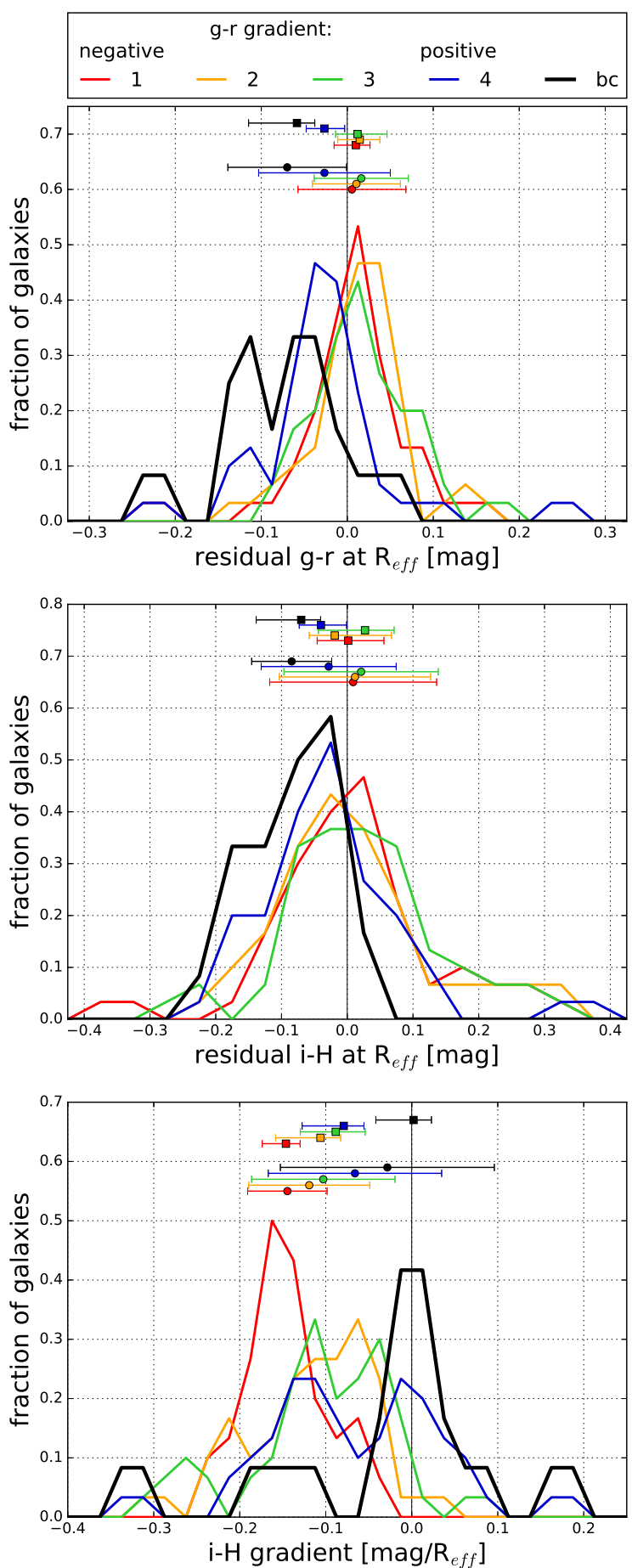

Fig. 4. Distribution of $(g-r)_{R_{\mathrm{eff}}}$ residual colour $(t o p),(i-H)_{R_{\mathrm{eff}}}$ residual colour (middle), and $i-H$ gradient (bottom), shown separately for each quartile in $g-r$ gradient. These quartiles are $1=[-0.100,-0.032]$, $2=[-0.031,-0.007], 3=[-0.004,0.025]$ and $4=[0.028,0.384]$ in $\mathrm{mag} / R_{\mathrm{eff}}$. In addition to the four quartiles, the blue-cored early-type dwarfs that we define in Sect. 4.1 are shown as black curve. They are also included in the highest quartile (blue curve), i.e. they enter the plot twice. Each curve is normalized to an area of 1 and is drawn by connecting points in steps of $0.05 \mathrm{mag}$ along the $x$-axis $(0.025 \mathrm{mag}$ for the top panel). Each point is the normalized number of galaxies within $\pm 0.05 \mathrm{mag}( \pm 0.025 \mathrm{mag}$ for the top panel). The coloured circles and horizontal bars indicate the respective mean and standard deviation of the distributions, whereas the coloured squares give median and the first and third quartile of the distributions. The uncertainty of the mean value is smaller by a factor of $\sqrt{30}$ (and by a factor of $\sqrt{12}$ for the blue-cored galaxies) than the displayed standard deviations.
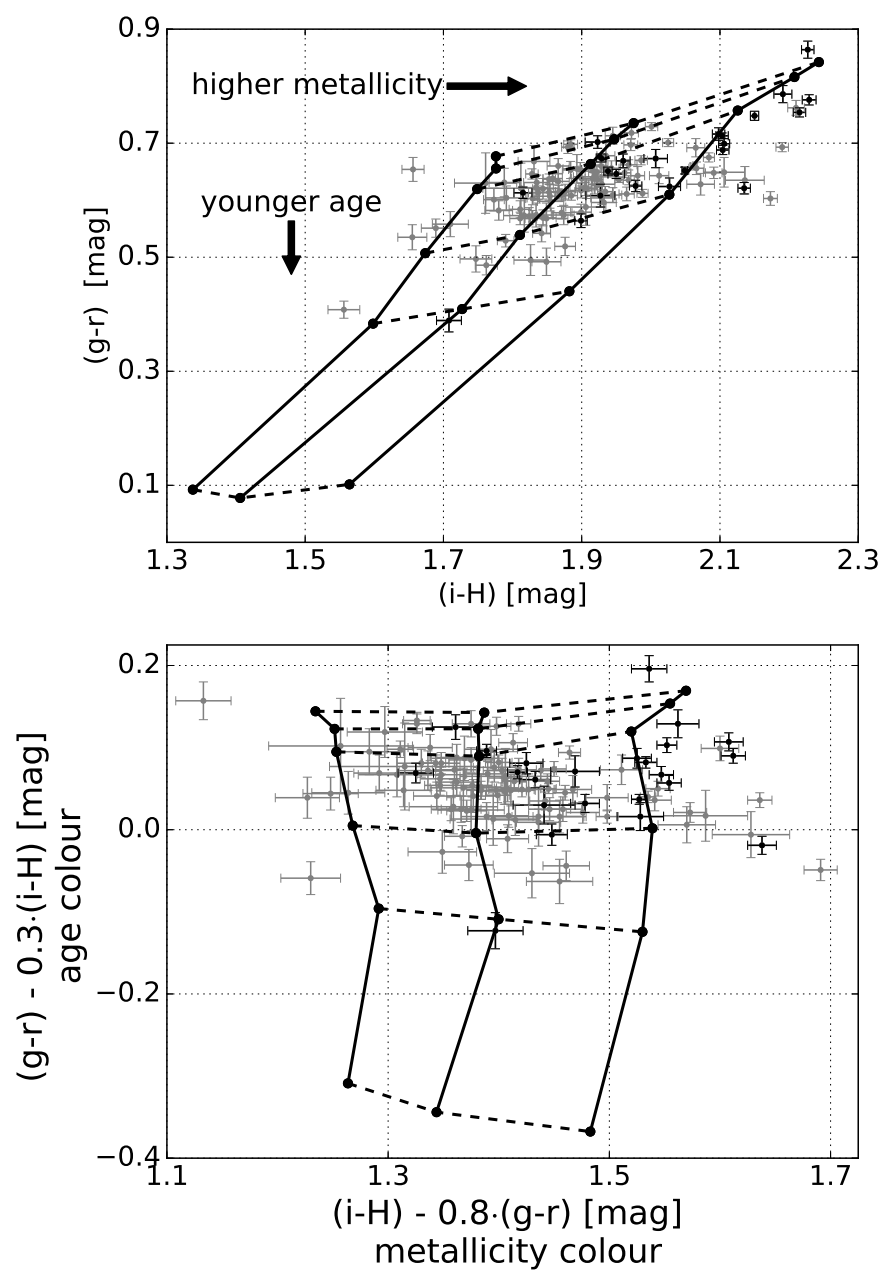

Fig. 5. Stellar population model: based upon the population synthesis code of Bruzual \& Charlot (2003), the solid model tracks are curves of constant metallicity (from left to right): $Z=0.008, Z=0.004$, and $Z=$ 0.02 , and marked at ages (from bottom to top) of $1,3,4.5,7.5,10.5$, and 13.5 Gyr, where age means the time since the onset of star formation. Top panel: $i-H$ is plotted against $g-r$. Bottom panel: metallicity colour $[(i-H)-0.8 \cdot(g-r)]$ is plotted against age colour $[(g-r)-0.3 \cdot(i-H)]$. For comparison, we plot the galaxy colours measured at the effective radius, using grey for galaxies originally classified as dwarfs and black for those originally classified as non-dwarfs.

colour. Based on spectral energy distributions of model stellar populations, optical colours such as $g-r$ can be considered agesensitive, whereas near-infrared (NIR) colours such as $i-H$ can be considered metallicity-sensitive (see e.g. Bruzual \& Charlot 2003). Therefore, a bluer colour in $g-r$ would imply a younger stellar population, while a redder colour in $i-H$ would imply a more metal-enriched stellar population.

However, looking in more detail at the colours of a stellar population model (Fig. 5) that was generated using the population synthesis code of Bruzual \& Charlot (2003, hereafter $\mathrm{BC} 03)^{6}$, we find that the $g-r$ colour is mainly age-sensitive and a little bit metallicity-sensitive, whereas the $i-H$ colour is ageand metallicity-sensitive to similar degrees (Fig. 5, upper panel). Therefore, the slight correlation that we find between $g-r$ and $i-H$ gradients can be explained by age gradients that contribute

6 We use the low-resolution BaSeL 3.1 stellar library, Padova 1994 isochrones (Bertelli et al. 1994), a Chabrier initial mass function (Chabrier 2003), and an exponentially decaying burst of star formation with a decay time $\tau=1 \mathrm{Gyr}$. 

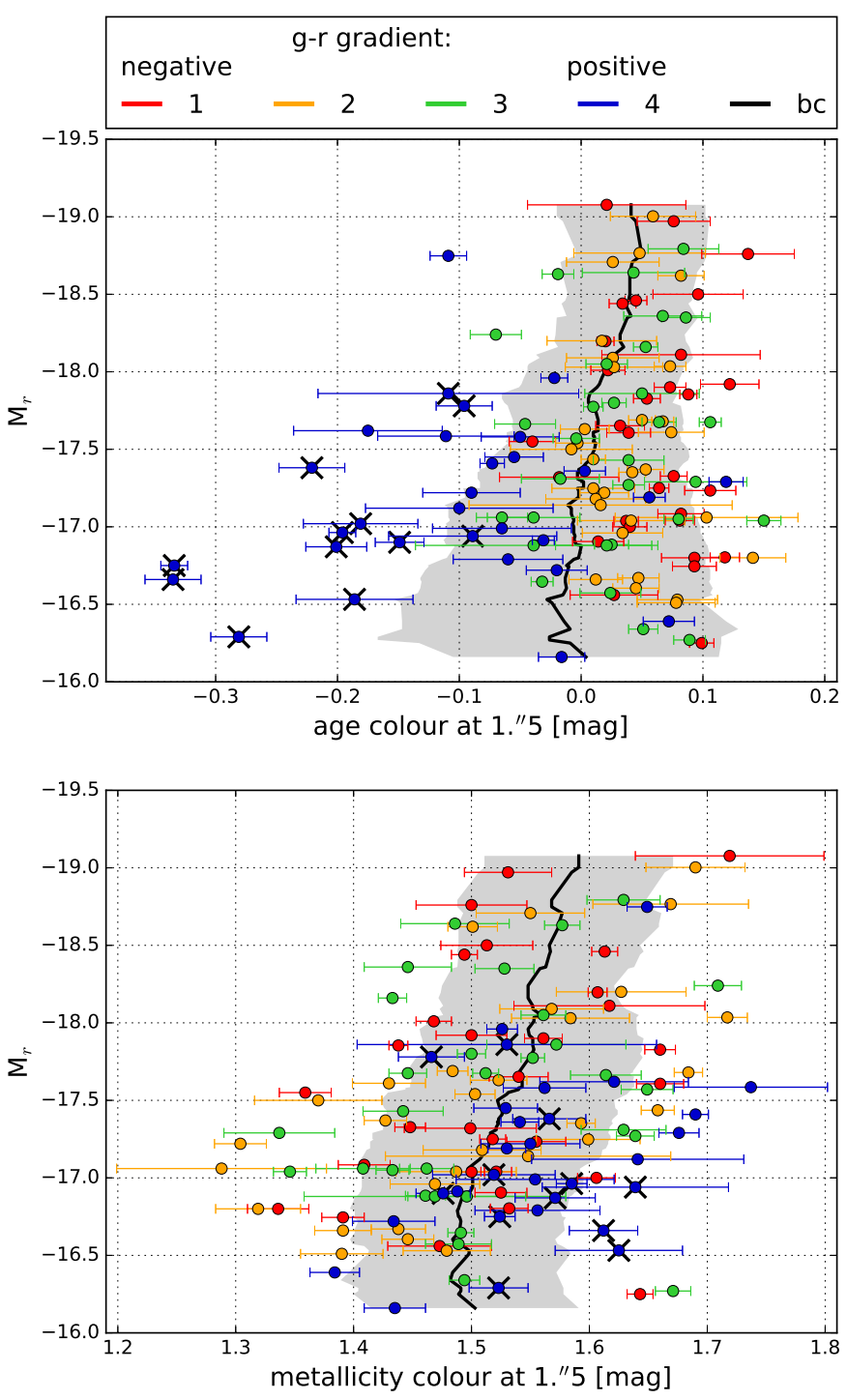

Fig. 6. Central colour magnitude relation: age colour measured at $11^{\prime \prime 5}$ (upper panel) and metallicity colour measured at 1".5 (lower panel) are plotted against the absolute $r$-band magnitude. Error bars denote the uncertainties of the isophotal colours. Galaxies are coloured based on their $g-r$ gradient groups (see Fig. 4). The black line describes the running mean of the colour within a 1 mag interval and the grey shaded area displays the corresponding standard deviation of the galaxies.

equally to both colours. The trend of $i-H$ gradients being more negative than the corresponding $g-r$ gradients can be explained by metallicity gradients that contribute more to $i-H$ than to $g-r$.

To generate an age-only and a metallicity-only colour in a simplified way, we subtract the estimated $i-H$ metallicity contribution from $g-r$ and the estimated $g-r$ age contribution from $i-H$ with appropriate factors (compare the upper and lower panels in Fig. 5). We thus define an approximate age-only colour as $[(g-r)-0.3 \cdot(i-H)]$ and an approximate metallicity-only colour as $[(i-H)-0.8 \cdot(g-r)]$; hereafter we refer to these as age colour and metallicity colour.

In Fig. 6 the central age colour (upper panel) and the central metallicity colour (lower panel) are plotted against the absolute $r$-band magnitude. Each galaxy is coloured based on its $g-r$ gradient group (same colouring as in Fig. 4). Galaxies with stronger positive $g-r$ gradients, especially blue-cored early-type dwarf galaxies, tend to have bluer age colours in the central region, but

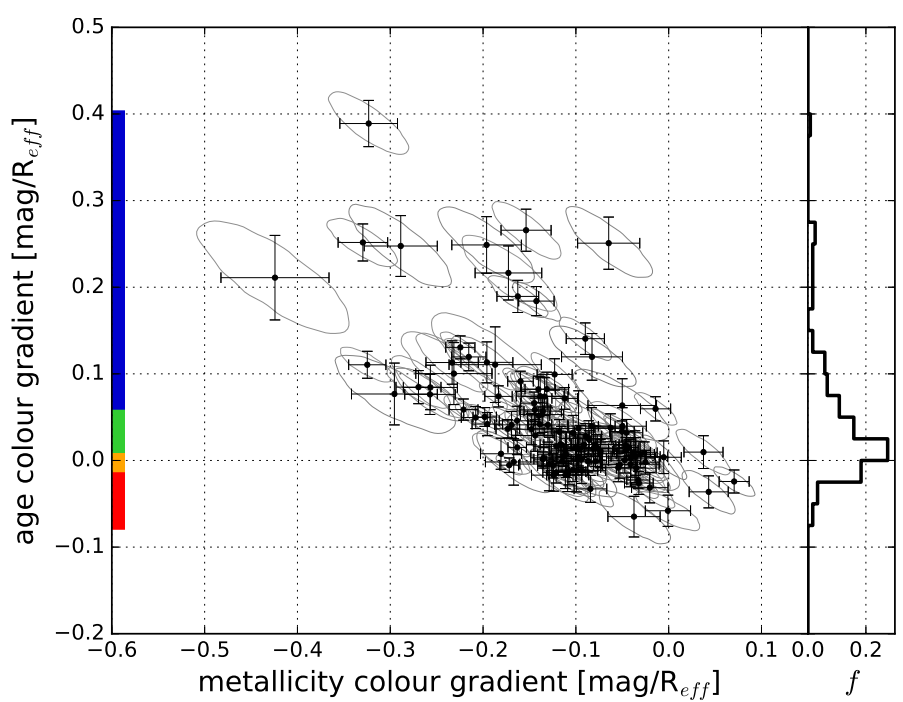

Fig. 7. Metallicity colour gradients of our galaxies plotted against their age colour gradients (see text for definitions). The error bars denote the standard errors (see Sect. 3). The correlation of the errors is displayed by a two-dimensional $1 \sigma$ Gaussian contour. The colour-coding on the $y$ axis defines the ranges of the four subsamples that all include the same number of galaxies (30). The fraction $f$ of galaxies in the age colour gradient distribution is presented in the right histogram.

otherwise we find almost no correlation between age colour and magnitude. The broad correlation of metallicity colour and magnitude reflects the well-known relation between stellar mass and metallicity (e.g. Barazza \& Binggeli 2002). To take into account these colour-magnitude relations, we computed residual age and metallicity colours (following the approach in Sect. 3): the difference between a galaxy's colour and the mean colour (black line in Fig. 6) of all sample galaxies within a \pm 0.5 mag interval around the galaxy is the residual age/metallicity colour that we use in the following.

Analogously, we can generate a simplified age colour gradient of $[\delta(g-r)-0.3 \cdot \delta(i-H)]$ and a simplified metallicity colour gradient of $[\delta(i-H)-0.8 \cdot \delta(g-r)]$. The resulting values are displayed in Fig. 7. We find that they are the same, within the errors, as when converting each galaxy's $g-r$ and $i-H$ colour profiles into age and metallicity colour profiles, and then fitting their gradients.

For the newly defined age/metallicity colours and colour gradients, we have to consider that we introduced correlations by construction and have therefore correlated errors. In Fig. 7 the correlated errors of the calculated age and metallicity colour gradients are displayed as a $1 \sigma$ Gaussian contour.

\subsection{Stellar populations}

In Fig. 8 the sample is divided into four equal subsamples based on their age colour gradients. The ranges of these subsamples are shown by the colour-coding in Fig. 7. The red subsample includes galaxies with negative age colour gradients (the lowest quartile) and the blue subsample includes the galaxies with the strongest positive age colour gradients (the highest quartile). The black subsample contains all quantitative blue-cored earlytype dwarf galaxies, as before, and all of them are also part of the blue subsample. For each subsample the distribution of the residual age colour measured at the effective radius is plotted in the upper panel of Fig. 8. We find no clear correlation between the age colour gradient and the residual (age colour) ${ }_{R_{\text {eff }}}$, but we 
find that the galaxies defined as quantitative blue-cored earlytype dwarf galaxies tend to have bluer residual (age colours) $R_{\text {eff }}$. This implies that blue-cored early-type dwarf galaxies tend to have a younger stellar population overall, and an even younger stellar population in their central region, since blue-cored earlytype dwarf galaxies possess strong age colour gradients.

In the middle panel of Fig. 8, where the distribution of the metallicity colour gradient is plotted for each subsample, we see that nearly all galaxies have negative metallicity colour gradients. Galaxies with a strong age colour gradient also tend to have a strong metallicity colour gradient. Furthermore, we find that the galaxies with the strongest age colour gradients possess the reddest central residual metallicity colours (Fig. 8, lower panel). These results imply metal-enriched central regions for almost all early-type dwarf galaxies and, at first glance, that galaxies with strong age colour gradients also have the strongest metallicity gradients and the most metal-enriched stellar populations in their centre.

However, we have to consider that colours and colour gradients are luminosity weighted. Therefore, we need to consider a more realistic stellar composition that consists of a younger and an older population.

To compare our colour gradient values with synthetic colour gradients modelled from composite stellar populations we used the BC03 library. Following the simplified approach of Lisker et al. (2006b) of using single-age populations, we assume an old population of $7 \mathrm{Gyr}$ and $Z=0.004$ for the whole galaxy (estimated from Fig. 5) and an additional young population that makes up $5 \%$ of the local present-day stellar mass in the centre (discussed in Sect. 5.1). We varied age and metallicity of the young population and calculated the colour difference between the old population (which represents the situation at $R_{\mathrm{eff}}$ ) and the composite population (which represents the situation at the galaxy centre). We use these calculated differences as model gradients and compare them to our fitted gradients (Fig. 9). Each model track in the figure has a single value for the age gradient, with the age of the young population given in the legend. The tracks are marked at different metallicity gradients, with the metallicity of the young population from left to right: $Z=0.1$, $Z=0.05, Z=0.02, Z=0.008$, and $Z=0.004$. Therefore, the rightmost point of a model track represents an age-only gradient and the leftmost point represents the largest metallicity gradient in addition to the age gradient.

We find that a stellar population younger than $500 \mathrm{Myr}$ is needed to match the measured colour gradients of the blue-cored early-type dwarf galaxies in addition to assuming a mean age of $7 \mathrm{Gyr}$ and a mean metallicity of $Z=0.004$. When we increase the present-day mass ratio of the young population up to $9 \%$ we can reproduce the blue-cored early-type dwarfs' colour gradients with a young population of up to $700 \mathrm{Myr}$. Furthermore, when we increase the mean age to $9 \mathrm{Gyr}$, the young population has to be younger than $600 \mathrm{Myr}$, and when we decrease the mean age to $5 \mathrm{Gyr}$, we need a young population younger than $400 \mathrm{Myr}$ to cover the locus of blue-cored early-type dwarfs in Fig. 9. Additionally we find that most blue-cored early-type dwarf galaxies, as well as most normal early-type dwarfs, have a central stellar population with supersolar metallicity. This, however, does not imply a supersolar mean metallicity since the central metal

7 Lisker et al. (2006b) assumed that the old population makes up at least $90.9 \%$ and the young population less than $0.3 \%$ of the total mass, except for VCC 1499 for which they assume that the old population makes up $75 \%$ and the young population $0.8 \%$.
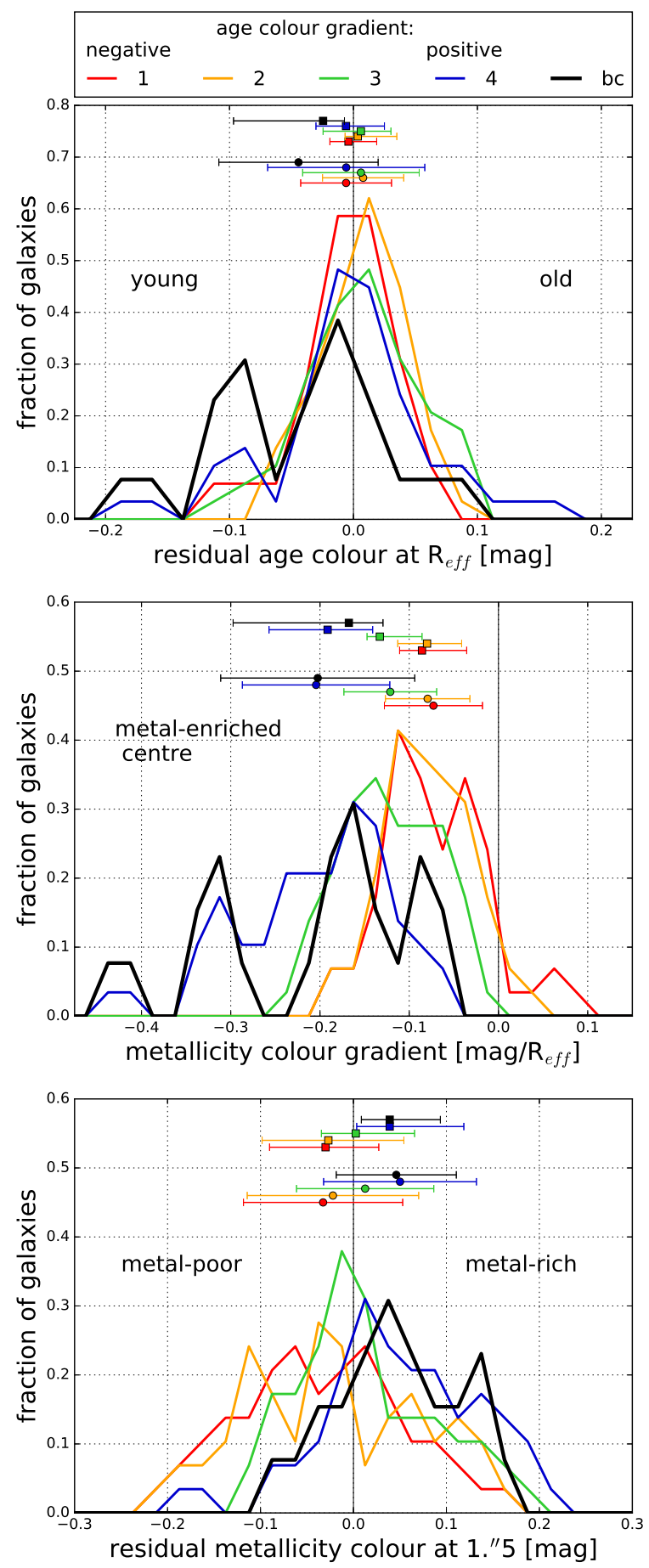

Fig. 8. Distribution of residual age colour (top), metallicity colour gradient (middle), and residual metallicity colour (bottom), shown separately for each quartile in age colour gradient. These quartiles are $1=[-0.065,0.001], 2=[0.002,0.019], 3=[0.024,0.072]$ and $4=$ $[0.074,0.389]$ in $\mathrm{mag} / R_{\text {eff }}$. In addition to the four quartiles, the bluecored early-type dwarfs that we define in Sect. 4.1 are shown as a black curve. They are also included in the highest quartile (blue curve), i.e. they enter the plot twice. Each curve is normalized to an area of 1 and is drawn by connecting points in steps of 0.05 mag along the $x$-axis. Each point is the normalized number of galaxies within $\pm 0.05 \mathrm{mag}$. The coloured circles and horizontal bars indicate the respective mean and standard deviation of the distributions, whereas the coloured squares give median and the first and third quartile of the distributions. The uncertainty of the mean value is smaller by a factor of $\sqrt{30}$ (and by a factor of $\sqrt{12}$ for the blue-cored galaxies) than the displayed standard deviations. 
enriched (young) stellar population makes up only a small fraction of the total mass.

As mentioned previously, we have to consider that colour gradients are luminosity weighted. The younger the young population, the larger its contribution to the galaxy's central colour and therefore, the stronger the colour gradients. This explains why, in Fig. 9, the vertical steps between the model tracks become larger with decreasing age of the young population, even though the step in the age gradient itself is always exactly $100 \mathrm{Myr}$. It also explains why the tracks become longer horizontally, even though the difference in the young population's metallicity between the left and right ends of the tracks is always the same.

In summary, we can say that almost all early-type dwarf galaxies have non-zero metallicity gradients and that the metallicity gradients of blue-cored early-type dwarfs are consistent with those of normal early-type dwarfs.

\section{Discussion}

\subsection{Stellar content}

Our colour analysis indicates that the stellar content of bluecored early-type dwarf galaxies can be explained by a young stellar population that makes up a small fraction of the mass in the central region. Therefore, blue-cored early-type dwarf galaxies are similar to normal early-type dwarfs, but have an additional young stellar population, or star forming region, in the centre. This is confirmed by the presence of prominent Balmer absorption in most cases and $\mathrm{H} \alpha$-emission in some cases, as noted by Lisker et al. (2006b) from SDSS spectra of the inner $1^{\prime \prime .5}$ (in radius). These authors modelled ${ }^{8}$ the spectra of blue-cored early-type dwarfs with a composite stellar population made up of three simple stellar populationss: an old (5 Gyr), an intermediate-age (10 Myr to $1 \mathrm{Gyr}$ ), and a young ( $<10 \mathrm{Myr}$ ) stellar population. Furthermore they assumed the same metallicity $(Z=0.004)$ for all populations.

However, their best-fitting composite populations would have systematically bluer $i-H$ colours and systematically more positive $i-H$ gradients than our measured values. The $g-r$ colour and colour gradients would be in the same overall range, but with a tendency toward slightly bluer colours and more positive gradients. These apparent discrepancies in $i-H$ can be explained by the lack of a metallicity difference between the old and young populations of Lisker et al. (2006b). When assuming a higher metallicity for the young and maybe the intermediateage population we get redder $i-H$ colours and weaker $i-H$ gradients. Additionally, we have to consider that Lisker et al. investigated only the innermost region of 1'.5 with their spectral analysis, while we do not examine this central part in our colour analysis. Since we found that blue-cored early-type dwarfs possess strong age gradients, it is possible that Lisker et al. observed a very young stellar population in the central part that our analysis is not sensitive to.

Additionally, Lisker et al. (2006b) mentioned that varying the ages of the different populations has a similar effect on the composite spectrum to changing the ratio of the mass fractions. We find the same result for the colours and colour gradients. When we assume an old population of 7 Gyr that makes up $95 \%$

\footnotetext{
8 They used the BC03 library with high spectral resolution, which was produced using the Bruzual \& Charlot (2003) Galaxy Isochrone Spectral Synthesis Evolution Library (GISSEL) code, implementing the Padova 1995 isochrones (Fagotto et al. 1994; Girardi et al. 1996) combined with the STELIB (Le Borgne et al. 2003) stellar library.
}
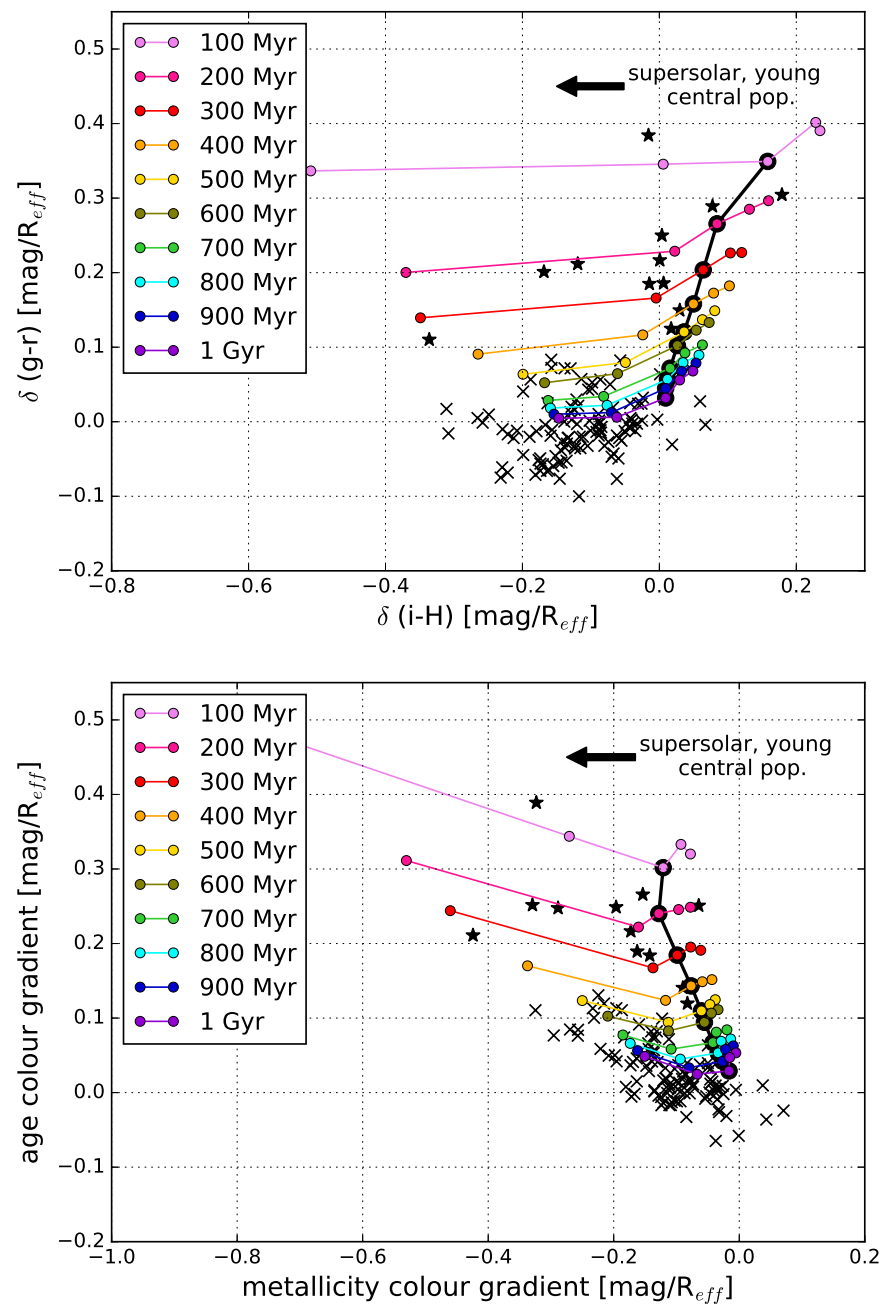

Fig. 9. Composite stellar population model, based upon the population synthesis code of Bruzual \& Charlot (2003). Each model track has a single value for the age gradient (old pop.: $7 \mathrm{Gyr}, Z=0.004$; composite pop.: $95 \%$ old and 5\% young; age of young pop. in the label) and is marked at different metallicity gradients (metallicity of the young pop. From left to right: $Z=0.1, Z=0.05, Z=0.02, Z=0.008$, and $Z=0.004)$. The black solid line denotes solar metallicity for the young population. Blue-cored early-type dwarf galaxies are displayed as asterisks.

of the total mass and a young population of $200 \mathrm{Myr}$, the colours and gradients are similar to when we assume instead an old population of 7 Gyr that only makes up $91 \%$ of the total mass and a young population of $300 \mathrm{Myr}$, or an old population of $9 \mathrm{Gyr}$ that makes up $95 \%$ of the total mass and a young population of 300 Myr.

Therefore, it is not possible to determine the exact stellar content of blue-cored and normal early-type dwarf galaxies, especially as we only perform a colour analysis and do not have spectra of different regions within a galaxy. Nevertheless, we can conclude that normal early-type dwarf galaxies have a mean age of about 5-9 Gyr and a subsolar mean metallicity within the framework of Bruzual \& Charlot's stellar population models, as described in Sect. 4.2. This is consistent with the results of e.g. Geha et al. (2003) and Van Zee et al. (2004a) who found in their spectral analysis a mean age of 5-7 Gyr and clearly subsolar metallicities, and the results of Michielsen et al. (2008) and Toloba et al. (2014b) who found a mean age of about 6 Gyr and a mean metallicity of $\log \left(Z / Z_{\odot}\right) \approx-0.6$. Roediger et al. (2011) 
found a somewhat older mean age (9-10 Gyr) of dwarf ellipticals from a different data set of optical-NIR photometry. When we subdivide our sample of faint early-type galaxies into galaxies originally classified as dwarfs and non-dwarfs, we find a similar age range for both, but a trend for non-dwarfs to be a bit more metal-rich than the dwarfs (see Fig. 5). This is consistent with the findings of Guérou et al. (2015).

We can infer that blue-cored early-type dwarf galaxies have mean ages and metallicities within the range of normal faint early-type galaxies, and are not separated from them. They do, however, belong to those galaxies with an overall younger stellar age. They feature an additional stellar population in the central region that has supersolar metallicity and is younger than $500 \mathrm{Myr}$, which implies that a blue-cored early-type dwarf galaxy can become a normal early-type dwarf within $0.5 \mathrm{Gyr}$, if no further central star formation occurs. In the literature it is often said that early-type dwarf galaxies have solar or subsolar metallicities (e.g. Van Zee et al. 2004b). However, Gu et al. (2006) found a high-metallicity HII region in the blue-cored dwarf elliptical galaxy IC 225 and Peeples et al. (2008) found some low-mass galaxies with high central gas metallicity and blue cores. Peeples et al. (2008) predicted that if a galaxy has a relatively low gas fraction, a small amount of pollution is able to enrich the gas. They suggested that these galaxies are transition types from gas-rich dwarf irregulars to gas-deficient dwarf ellipticals, even though it remains unclear whether a significant fraction of stars would still be able to form from the enriched gas. However, supersolar metallicities in faint early-type galaxies are already observed and are not inconsistent with a mean subsolar metallicity, since the high-metallicity portion makes up only a small fraction in mass.

In addition, we found that nearly all early-type dwarfs possess negative metallicity gradients, which has also been found by Sybilska et al. (2017), and across all galaxy types in the Virgo Cluster (e.g. Roediger et al. 2011). The lower panel of Fig. 5 indicates that a metallicity difference of about $0.7 \mathrm{dex}$ (left to right model track) implies a colour difference of more than 0.2 mag in metallicity colour, while a metallicity difference of 0.3 dex (left to middle model track) implies a colour difference in metallicity colour of about $0.1 \mathrm{mag}$. Since we find that most early-type dwarf galaxies possess a metallicity colour gradient of about $-0.1 \mathrm{mag} / R_{\text {eff }}$ and almost all blue-cored early-type dwarf galaxies a metallicity colour gradient $\lesssim-0.1 \mathrm{mag} / R_{\text {eff }}$, our results are consistent with the average value of $-0.5 \mathrm{dex} / R_{\mathrm{eff}}$ for dwarf elliptical galaxies in the Fornax cluster and nearby groups of galaxies found by Koleva et al. (2009). The metallicity gradient for the Local Group dwarf elliptical galaxy NGC 205 of $-0.6 \mathrm{dex} / R_{\mathrm{eff}}$ found by Koleva (2009) and the values for Virgo cluster earlytype dwarf galaxies found by Chilingarian (2009, his Table 3) are also consistent with our results.

In our whole analysis we neglected dust extinction, which in principle could cause redder colours. If dust had a significant effect on our galaxies' colours, we should find the flattest galaxies to be redder, which is not the case; in fact, we find that the roundest galaxies tend to be redder in $i-H$. Additionally, Conselice et al. (2003), di Serego Alighieri et al. (2007), and De Looze et al. (2010) showed that early-type dwarf galaxies include little to no dust. De Looze et al. argued that the removal of interstellar dust from dwarf galaxies has to be as efficient as the removal of interstellar gas. Nonetheless, VCC 0209, the galaxy with the strongest metallicity colour gradient $\left(-0.42 \mathrm{mag} / R_{\mathrm{eff}}\right)$ has been detected with WISE (Boselli et al. 2014) and Herschel (Auld et al. 2013, in all five bands) and therefore contains a significant amount of dust.
Another factor which has to be considered in blue-cored galaxies is the projection effect. Ignoring any gas and dust content we can make a simple estimate for the integrated amount of light that we receive along a given line of sight. We adopt an exponential profile and consider a flattened dwarf galaxy with an effective radius of $1.2 \mathrm{kpc}$ along the major axis (median size of our blue-cored dwarf sample) and an axis ratio of 0.45 (which is approximately the intrinsic axis ratio of blue cored early-type dwarfs that was inferred by Lisker et al. 2007). We assume that the galaxy includes a young population that is confined to an inner region with a semi-major axis of $0.4 \mathrm{kpc}$ and an axis ratio of 0.85 , i.e. rounder than the main body of the galaxy. We now compare the situation when observing this galaxy along its minor and its major axes, with a line of sight through the galaxy centre. The light fraction $f$ that we would receive from the inner region along the minor axis $(f=0.65)$ is 1.5 times larger than the fraction we would get along the major axis $(f=0.43)$. This factor increases to 1.7 if we halve the size of the inner region; it decreases to 1.4 if we flatten the inner region to an axis ratio of 0.7 . If we now assume that the young population makes up $7.7 \%$ of the present-day stellar mass of the inner region, it would contribute $5.0 \%$ to the light along the minor axis and $3.3 \%$ along the major axis. A $g-r$ gradient that we would measure to be $0.10 \mathrm{mag} / R_{\text {eff }}$ when observed along the minor axis would therefore be measured to be only $0.075 \mathrm{mag} / R_{\text {eff }}$ when observed along the major axis. In principle, this could lead to missing detections of quantitative blue-cored early-type dwarf galaxies, which we defined as having more than $0.10 \mathrm{mag} / R_{\text {eff }}$. However, in our sample there are only two galaxies, VCC 0165 and VCC 1049, with gradients in this range.

\subsection{Formation scenarios}

It has often been suggested that early-type dwarf galaxies are remnants of infalling late-type galaxies that are modified by the strong forces acting within a cluster environment (e.g Van Zee et al. 2004b; Boselli et al. 2008; De Rijcke et al. 2010; Toloba et al. 2014a) and that blue-cored early-type dwarf galaxies may be transition types between late-type galaxies and earlytype dwarfs (e.g. Lisker et al. 2006b). A strong age gradient with a younger, metal-enriched central region could in principle have been generated by quenching star formation only in the outskirts and/or by increasing the star formation rate in the centre. Additionally, if part of the early-type dwarf galaxies had joined the Virgo cluster more recently, we would also expect a younger mean age. This is exactly what we find for the blue-cored earlytype dwarf galaxies.

The two most often suggested scenarios are ram pressure stripping (Gunn \& Gott 1972) and galaxy harassment (Moore et al. 1996, 1998); the latter can be split into two components, tidal shocking with a short timescale as in high-speed, massive encounters and tidal heating with a long timescale as for galaxies that pass the central cluster's potential well closely (Smith et al. 2010). Moore et al. (1998) showed in their simulations that galaxy harassment causes the gas to funnel to the centre and form a gas density excess. However, it remained unclear whether this would lead to blue cores, as the authors remarked that feedback could halt the collapse of the gas. Moreover, Smith et al. (2010) found in a Monte Carlo simulation of galaxy harassment by a Virgo-like harasser population that strong tidal encounters are very rare, involving less than $15 \%$ of the infalling dwarfs. Furthermore, Smith et al. (2015) pointed out that although their model galaxies spent many Gyrs in the cluster environment, most dwarf galaxies showed no stripping or 
change in radial distribution of stars. Hence, it has not been ruled out that galaxies are influenced by harassment, but if galaxy harassment is able to trigger star formation it would only be efficient for a small fraction of the dwarf galaxies in a Virgo-like cluster, and those would be in a range of different evolutionary states at present. It should be noted, however, that the slower tidal interactions typical of group environments were found to be able to induce gas infall and cause central starbursts in simulations of low-mass disk galaxies (Yozin \& Bekki 2015). This may be of relevance if the Virgo cluster blue-cored early-type dwarf galaxies - possibly an infalling population (Lisker et al. 2006b) - had resided in groups until recently and were pre-processed there (e.g. De Lucia et al. 2012).

There is more agreement with the ram pressure stripping scenario, where the interstellar medium (ISM) of a late-type galaxy is removed by the hot intracluster medium (ICM) as it flows past. For example, Conselice et al. (2001) argued that the Virgo cluster dwarfs are an infall population and Boselli et al. (2008) demonstrated that ram-pressure stripping can play a prominent role for the star formation histories of dwarf galaxies if gas is removed quickly. Furthermore, De Rijcke et al. (2010) showed that some observational quantities in the Fornax cluster could be explained by assuming late-type dwarf galaxies falling into the cluster and evolving into early-type dwarf galaxies by ram pressure stripping. Mori \& Burkert (2000) found that for galaxies with masses below $10^{9} M_{\odot}$, which is roughly the mass of most blue-cored early-type dwarf galaxies, the ram pressure of the ICM in a typical cluster environment exceeds the gravitational force of the galaxy. Thus their gas gets removed entirely, but for more massive galaxies, the gas can survive in the central region of the gravitational potential well. Therefore, the gas in the outer regions of the infalling galaxy can be stripped by the ICM, while the gas in the inner part remains. It can be shown that the Virgo intracluster medium (Vollmer 2009) would not be able to remove gas from the centres of most quantitative blue-cored early-type dwarf galaxies ${ }^{9}$. Thus, the galaxy continues to have active star formation in the central region - at a rate that may even be increased due to ram pressure (Kronberger et al. 2008) - while the outer parts are reduced to a passively evolving stellar population. Such a development clearly implies an age gradient and, if the central region retains a large fraction of its enriched material, also a metal-enriched centre. Since this is exactly what we find, our colour analysis corroborates the idea of ram pressure stripping.

It remains to be mentioned that it is also possible that some early-type dwarf galaxies do not originate from infalling galaxies and that not all early-type dwarfs have necessarily been bluecored early-type dwarfs at some point in the past. To get a better insight into their evolution, it is important to have larger samples of spectroscopic $[\alpha / \mathrm{Fe}]$ abundances for low-mass earlytype galaxies in order to understand how the (early) environment governed the star formation timescales of low-mass galaxies

\footnotetext{
9 At a clustercentric distance of $0.5 \mathrm{Mpc}$ the density of the Virgo intracluster medium as modelled by Vollmer (2009) provides a ram pressure of $10^{-11.13} \mathrm{~N} / \mathrm{m}^{2}$ when assuming a relative velocity of $1000 \mathrm{~km} \mathrm{~s}^{-1}$, and a ram pressure of $10^{-11.32} \mathrm{~N} / \mathrm{m}^{2}$ at a clustercentric distance of $1 \mathrm{Mpc}$. We use ugriz photometry to approximate the stellar surface mass density (based on Hansson et al. 2012 at one exponential scale length) for all quantitative blue-cored early-type dwarf galaxies in the Virgo cluster. When assuming a gas surface density of one-tenth of the stellar surface density we obtain estimates for the restoring force per unit area between $10^{-11.94}$ and $10^{-10.76} \mathrm{~N} / \mathrm{m}^{2}$ and between $10^{-11.08}$ and $10^{-9.89} \mathrm{~N} / \mathrm{m}^{2}$ when extrapolating these values to the galaxies' centres, using an exponential profile.
}

(Michielsen et al. 2008; Liu et al. 2016), and hence their stellar population build-up. From simulations, Schroyen et al. (2011) showed that the development of metallicity gradients is anticorrelated with angular momentum (see also Leaman et al. 2013). Accurate measurements of stellar age and metallicity gradients (Sybilska et al. 2017) in conjunction with the angular momentum distribution from integral-field spectroscopic data (Ryś et al. 2014; Guérou et al. 2015) are essential for early-type dwarf galaxy samples of significant size over a range of environments and, ideally, stellar mass.

Acknowledgements. T.L. and R.F.P. acknowledge financial support from the European Union's Horizon 2020 research and innovation programme under the Marie Skłodowska-Curie grant agreement No. 721463 to the SUNDIAL ITN network. G.v.d.V., R.L., A.S., R.F.P. and J.F.-B. acknowledge support from grant AYA2016-77237-C3-1-P from the Spanish Ministry of Economy and Competitiveness (MINECO). Paudel S. acknowledges the support from the Samsung Science \& Technology Foundation under Project Number SSTF-BA1501-0.

\section{References}

Adelman-McCarthy, J. K., Agüeros, M. A., Allam, S. S., et al. 2006, ApJS, 162, 38

Adelman-McCarthy, J. K., Agüeros, M. A., Allam, S. S., et al. 2007, ApJS, 172, 634

Auld, R., Bianchi, S., Smith, M. W. L., et al. 2013, MNRAS, 428, 1880

Barazza, F. D., \& Binggeli, B. 2002, A\&A, 394, L15

Barazza, F. D., Binggeli, B., \& Jerjen, H. 2002, A\&A, 391, 823

Barazza, F. D., Jogee, S., Rix, H.-W., et al. 2006, ApJ, 643, 162

Barazza, F. D., Wolf, C., Gray, M. E. et al. 2009, A\&A, 508, 665

Bertelli, G., Bressan, A., Chiosi, C., et al. 1994, A\&AS, 106, 275

Bertin, E., \& Arnouts, S. 1996, A\&AS, 117, 393

Binggeli, B., \& Cameron, L. M. 1991, A\&A, 252, 27

Binggeli, B., Sandage, A., \& Tammann, G. A. 1985, AJ, 90, 1681

Binggeli, B., Tammann, G. A., \& Sandage, A. 1987, AJ, 94, 251

Binggeli, B., Sandage, A., \& Tammann, G. A. 1988, ARA\&A, 26, 509

Boselli, A., Boissier, S., Cortese, L., \& Gavazzi, G. 2008, ApJ, 674, 742

Boselli, A., Voyer, E., Boissier, S., et al. 2014, A\&A, 570, A69

Bruzual, G., \& Charlot, S. 2003, MNRAS, 344, 1000

Chabrier, G. 2003, PASP, 115, 763

Chilingarian, I. V. 2009, MNRAS, 394, 1229

Conselice, C. J., Gallagher, J. S., \& Wyse, R. F. G. 2001, ApJ, 559, 791

Conselice, C. J., O’Neil, K., Gallagher, J. S., \& Wyse, R. F. G. 2003, ApJ, 591, 167

Dabringhausen, J., \& Kroupa, P. 2013, MNRAS, 429, 1858

Dabringhausen, J., Hilker, M., \& Kroupa, P. 2008, MNRAS, 386, 864

De Looze, I., Baes, M., Zibetti, S., et al. 2010, A\&A, 518, L54

De Lucia, G., Weinmann, S., Poggianti, B. M., Aragón-Salamanca, A., \& Zaritsky, D. 2012, MNRAS, 423, 1277

Den Brok, M., Peletier, R. F., Valentijn, E. A., et al. 2011, MNRAS, 414, 3052

De Rijcke, S., Dejonghe, H., Zeilinger, W. W., \& Hau, G. K. T. 2003, A\&A, 400, 119

De Rijcke, S., van Hese, E., \& Buyle, P. 2010, ApJ, 724, L171

di Serego Alighieri, S., Gavazzi, G., Giovanardi, C., et al. 2007, A\&A, 474, 851 di Serego Alighieri, S., Bianchi, S., Pappalardo, C., et al. 2013, A\&A, 552, A8

Fagotto, F., Bressan, A., Bertelli, G., \& Chiosi, C. 1994, A\&AS, 105, 39

Ferrarese, L., Côté, P., Sánchez-Janssen, R., et al. 2016, ApJ, 824, 10

Geha, M., Guhathakurta, P., \& van der Marel, R. P. 2003, AJ, 126, 1794

Girardi, L., Bressan, A., Chiosi, C., Bertelli, G., \& Nasi, E. 1996, A\&AS, 117, 113

Glass, L., Ferrarese, L., Côté, P., et al. 2011, ApJ, 726, 31

Graham, A. W., \& Guzmán, R. 2003, AJ, 125, 2936

Grebel, E., Gallagher, J. S., \& Harbeck, D. 2003, AJ, 125, 1926

Gu, Q., Zhao, Y., Shi, L., Peng, Z., \& Luo, X. 2006, AJ, 131, 806

Guérou, A., Emsellem, E., McDermid, R. M., et al. 2015, ApJ, 804, 70

Gunn, J. E., \& Gott, J. R. I. 1972, ApJ, 176, 1

Hansson, K. S. A., Lisker, T., \& Grebel, E. K. 2012, MNRAS, 427, 2376

Hodge, P. W. 1973, ApJ, 182, 671

Janz, J., \& Lisker, T. 2008, ApJ, 689, L25

Janz, J., Laurikainen, E., Lisker, T., et al. 2012, ApJ, 745, L24

Janz, J., Laurikainen, E., Lisker, T., et al. 2014, ApJ, 786, 105

Janz, J., Penny, S. J., Graham, A. W., et al. 2017, MNRAS, 468, 2850

Jerjen, H., Kalnajs, A., \& Binggeli, B. 2000, A\&A, 358, 845

Kim, S., Rey, S.-C., Lisker, T., \& Sohn, S. T. 2010, ApJ, 721, L72 
Kim, S., Rey, S.-C., Bureau, M., et al. 2016, ApJ, 833207

Koleva, M. 2009, Ph.D. Thesis, Univ. Lyon

Koleva, M., De Rijcke, S., Prugniel, P., \& Zeilinger, W. W. 2009, MNRAS, 396, 2133

Kormendy, J., Fisher, D. B., Cornell, M. E., \& Bender, R. 2009, ApJS, 182, 216 Kronberger, T., Kapferer, W., Ferrari, C., Unterguggenberger, S., \& Schindler, S. 2008, A\&A, 481, 337

Larson, R. B., Tinsley, B. M., \& Caldwell, C. N. 1980, ApJ, 237, 692

Leaman, R., Venn, K. A., Brooks, A. M., et al. 2013, ApJ, 767, 131

Le Borgne, J. F., Bruzual, G., Pelló, R., et al. 2003, A\&A, 402, 433

Liu, Y., Peng, E. W., Blakeslee, J., et al. 2016, ApJ, 818, 179

Lietzen, H., Tempel, E., Heinämäki, P., et al. 2012, A\&A, 545, A104

Lisker, T., Grebel, E. K., \& Binggeli, B. 2006a, AJ, 132, 497

Lisker, T., Glatt, K., Westera, P., \& Grebel, E. K. 2006b, AJ, 132, 2432

Lisker, T., Grebel, E. K., Binggeli, B., \& Glatt, K. 2007, ApJ, 660, 1186

Lisker, T., Grebel, E. K., \& Binggeli, B. 2008, AJ, 135, 380

Lisker, T., Weinmann, S. M., Janz, J., \& Meyer, H. T. 2013, MNRAS, 432, 1162

Michielsen, D., Boselli, A., Conselice, C. J., et al. 2008, MNRAS, 385, 1374

Moore, B., Katz, N., Lake, G., Dressler, A., \& Oemler, A. 1996, Nature, 379, 613

Moore, B., Lake, G., \& Katz, N. 1998, ApJ, 495, 139

Mori, M., \& Burkert, A. 2000, ApJ, 538, 559

Pak, M., Rey, S.-C., Lisker, T., et al. 2014, MNRAS, 445, 630

Paudel, S., Lisker, T., \& Kuntschner, H. 2011, MNRAS, 413, 1764

Peeples, M. S., Pogge, R. W., \& Stanek, K. Z. 2008, ApJ, 685, 904

Peletier, R. F. 1993, A\&A, 271, 51
Pierini, D. 2002, MNRAS, 330, 997

Poudel, A., Heinämäki, P., Nurmi, P., et al. 2016, A\&A, 590, A29

Poudel, A., Heinämäki, P., Tempel, E., et al. 2017, A\&A, 597, A86

Roberts, S., Davies, J., Sabatini, S., et al. 2007, MNRAS, 379, 1053

Roediger, J. C., Courteau, S., MacArthur, L. A., \& McDonald, M. 2011, MNRAS, 416, 1996

Roediger, J. C., Ferrarese, L., Côté, P., et al. 2017, ApJ, 836, 120

Ryś, A., van de Ven, G., \& Falcón-Barroso, J. 2014, MNRAS, 439, 284

Sandage, A., \& Binggeli, B. 1984, AJ, 89, 919

Schlegel, D. J., Finkbeiner, D. P., \& Davis, M. 1998, ApJ, 500, 525

Schombert, J. M. 2016, AJ, 152, 214

Schroyen, J., De Rijcke, S., Valcke, S., et al. 2011, MNRAS, 416, 601

Smith, R., Davies, J. I., \& Nelson, A. H. 2010, MNRAS, 405, 1723

Smith, R., Sánchez-Janssen, R., Beasley, M. A., et al. 2015, MNRAS, 454, 2502

Sybilska, A., Lisker, T., Kuntschner, H., et al. 2017, MNRAS, 470, 815

Toloba, E., Guhathakurta, P., van de Ven, G., et al. 2014a, ApJ, 783, 120

Toloba, E., Guhathakurta, P., Peletier, R. F., et al. 2014b, ApJS, 215, 17

Toloba, E., Guhathakurta, P., Boselli, A., et al. 2015, ApJ, 799, 172

Tully, R. B., \& Shaya, E. J. 1984, ApJ, 281, 31

Tully, R. B., \& Trentham, N. 2008, AJ, 135, 1488

Van Zee, L., Skillman, E. D., \& Haynes, M. P. 2004a, AJ, 128, 121

Van Zee, L., Barton, E. J., \& Skillman, E. D. 2004b, AJ, 128, 2797

Vazdekis, A., Ricciardelli, E., Cenarro, A. J., et al. 2012, MNRAS, 424, 157

Vigroux, L., Souviron, J., \& Vader, J. P. 1984, A\&A, 139, L9

Vollmer, B. 2009, A\&A, 502, 427

Yozin, C., \& Bekki, K. 2015, MNRAS, 453, 14 